\title{
UNIFIED TOPOLOGICAL FRAMEWORK FOR RETRIEVING 2D AND 3D MULTI-SCALE SPATIAL INFORMATION
}

\author{
Hairi Karim, Alias Abdul Rahman, Ali Jamali \\ 3D GIS Research Lab, \\ Faculty of Geoinformation and Real Estate, \\ Universiti Teknologi Malaysia, \\ 81310, Johor Bahru, Johor, Malaysia. \\ wmhairigis@gmail.com, alias@utm.my, and ali.jamali.65@gmail.com
}

KEY WORDS: Topological connectivity, CityGML 3D building, multi-scale LoD, 2D and 3D model

\begin{abstract}
:
Different applications or users need different model (differ requirement on level of details; geometry and information), less focuses in topological element. Improper manage geometry, attribute and topology elements in multi-scape environment will cause data repetition/redundancy, storage capacity and performance issues. This research focuses on utilizing topological graph which is important for various Geographical Information Science applications such as 3D indoor, network analysis, and sharing information/attribute from multiple level of details (LoD). A conceptual framework towards a unified data structure/model for representing several levels of details (LoDs) which integrates 2D and 3D topological and geometrical spaces in a single view is presented. The proposed framework has a potential of adopting into the CityGML/IndoorGML standards with representing different CityGML LoDs in a single view representation. Potential applications of the proposed conceptual framework including solar energy and utility mapping are discussed.
\end{abstract}

\section{INTRODUCTION}

Traditionally, 2D multi-scale objects are stored and structured separately in different databases and viewers. This situation creates some drawbacks especially in finding and getting information from other detailed objects - lack of relationships, limited queries and others. Thus, it produces limited information from the designed or developed applications.

For 3D cases, most of the application/user requires different abstraction details of the same model because of different needs, requirements, views and applications. Each application requires its own set of level of details (LoD) to be embedded into the model. Most of these models focus on geometry, attribute and semantic information; less focuses on topological element). This application/user-oriented customized models resulting difficulties for sharing with other stakeholders. To overcome this problem, Open Geospatial Consortium (OGC) introduced CityGML v1.0 in 2008 and v2.0 in 2011 as a standardised data model and exchange format for 3D models of city and landscape features.

CityGML solves only some of those sharing problems. However, representing the same 3D object (e.g. a building) with multiple representations as proposed by Löwner et. al (2016) may produces some major drawbacks; redundancy of other less focused element in research and real implementation (such as attribute and semantic information) and visualization (accessing only one model/LoD in a single viewer).

We strongly believe that a unified (2D and 3D seamless) geometry and topological data structure should be able to integrate information on multiple LoDs (CityGML) into a single viewer. Based on the above current situations, the proposed data structure could be utilized for future applications (3D mapping and analysis) including underground utility, 3D building management, 3D Cadastre, and other 3D applications for urban city planning.

Section 2 describes the topological modelling for 2D and 3D scale modelling, drawback of current implementation and lastly some previous works. Section 3 discusses on the proposed unify topological modelling framework while Section 4 describes on potential application such as underground utility network and estimation of solar panel. Conclusion of the paper will be in Section 5 .

\section{TOPOLOGICAL MODELLING IN SCALE DIMENSION}

\subsection{Scale Dimension}

Different applications/users need different abstractions of the real-world phenomenon (Karim et al., 2016); or in other words, different models of 2D/3D object. Each application requires its own set level of details (LoDs) to be embedded into the model. Most of the models focus on the geometry accuracy, attribute and some semantic information (visualization and measurement purposes). However, less emphasis was given on topological aspect such as for navigation purposes in 2D multi-scale or even 3D CityGML model.

For 2D cases, the current implementation frameworks of scale integration with 2D spatial data are either using the generalization techniques, storing the individual level of detail data into separate databases (multi-scale, Figure 1) or varioscale approaches (Figure 2). 


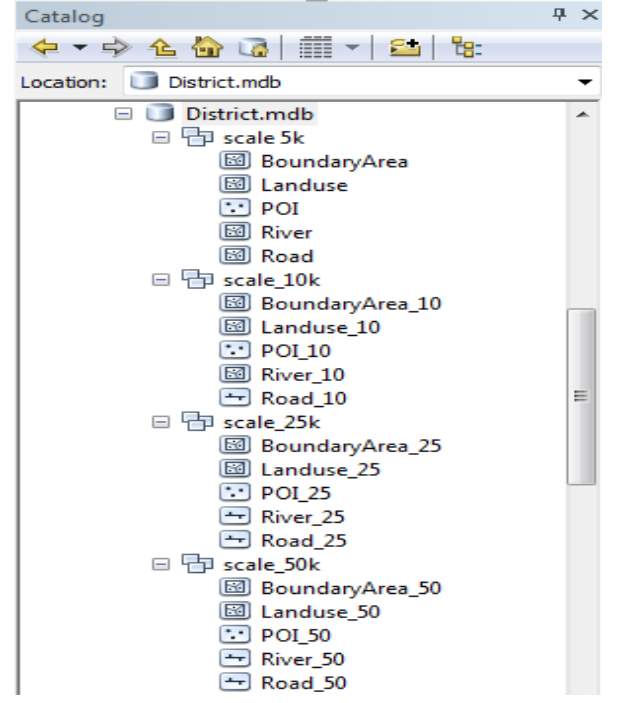

Figure 1: Multi-scale in separated database, with no connected topology between LoD and attribute redundancy.
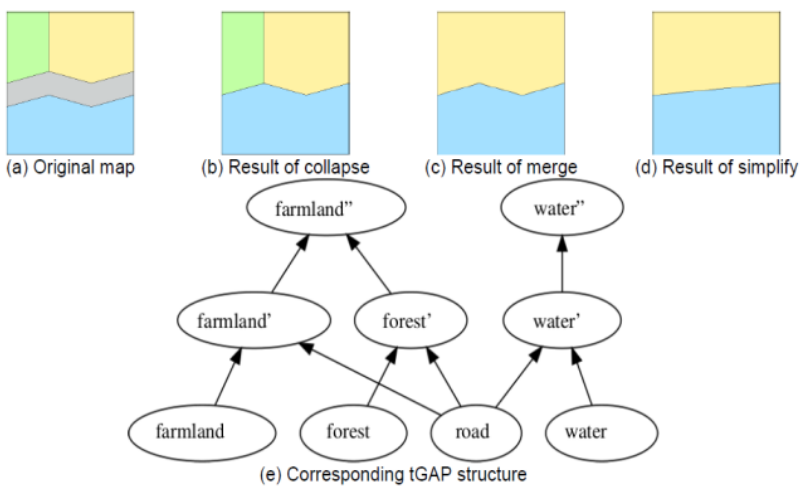

Figure 2: Vario-scale approach, topology connect hierarchical between LoD and good in storing attribute (Meijers, 2011).

For 3D GIS modelling, previous works implemented different generalization methods in order to produce less-detailed LoDs from the higher ones. Hiding less important details or/and reducing the data storage volume are available options for an efficient 3D analysis and visualization (Baig et al., 2011). The generalization processes (e.g. aggregation, simplification and other approaches) or a set of pre-defined level of details which are topologically disconnected to represent GIS model in scale dimension.

This application/user oriented customized models resulting difficulties in sharing the model to other stakeholders or receiving from others. Realizing these problems, Open Geospatial Consortium (OGC) has introduced CityGML v1.0.0 in 2008 and v2.0.0 (2011) as a standardised data model and exchange format for 3D models of city and landscape features. CityGML is an international standard for the representation and exchange of semantic 3D city and landscape models, which not only represents the shape and graphical appearance of city models but specifically addresses the object semantics and the representation of the thematic properties, taxonomies and aggregations (Groger and Plumer, 2011).
CityGML is an open data model using XML-based format for the storage and exchange of virtual city models. CityGML is a common information model for the representation of 3D urban objects. However, increasing professional applications give rise to needs of conceptual meanings beyond geometry since the pure appearance representation mainly focus on the photorealistic visualization while ignoring a full comprehension of the data. Numbers of applications like urban planning and facility supervision, disaster management and personal navigation require additional information, i.e. classification and relationship of components, about the city objects given in a standardized representation (Kwan and Lee, 2005). Therefore, the 3DCMs must incorporate the geometry and the semantics.

Existing datasets are often produced lacking semantics by using photogrammetric approach or CAD tools. An efficient way should be proposed to complement the thematic meanings of the geometry. At the same time, fundamental issues such as the consistency of geometry, semantics and topology were comprehensively studied (Kolbe et al., 2008; Kwan and Lee, 2005), which laid the basis of CityGML and semantic modeling. Recently, several extensions of CityGML are also proposed, such as the integration of both above and underground features as well as temporal semantics of house properties (Emgard and Zlatanova, 2008).

CityGML supports different Levels-of-Detail (LoD), which may occur from independent data collection processes and are used for well-organized visualization and efficient data analysis. In one CityGML data set, the same object may be presented in different LoD simultaneously, enabling the analysis and visualization of the same object with regards to various degrees of resolution/details. CityGML provides five different LoDs, which are illustrated in Figure 3.

LoD0 is essentially a two and a half dimensional Digital Terrain Model, over which an aerial image or a map may be draped. LoD1 is the well-known blocks model, without any roof structures or textures. Comparing LoD1 with LoD2, the latter has differentiated roof structures and textures and vegetation objects may also be represented. LoD3 denotes architectural models with detailed wall and roof structures, balconies, bays and projections. High-resolution textures can be mapped onto these structures. In addition, detailed vegetation and transportation objects are components of a LoD3 model. LoD4 completes a LoD3 model by adding interior structures like rooms, interior doors, stairs, and furniture.

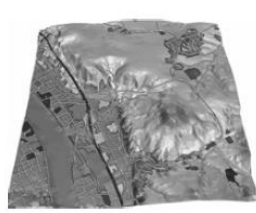

LoD0

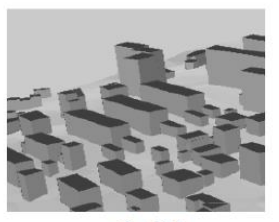

LoD1

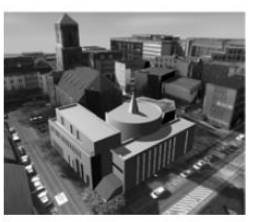

LoD2
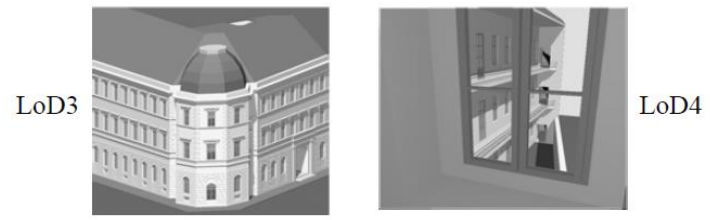

Figure 3. The five level of details (LoD) defined by CityGML 
CityGML is indeed solving some problems, but not entirely. There are many left-over focus elements need to be covered and standardized as more people started to realize the importance of spatial data and applications side is getting expended (more new applications). The level 4 of CityGML includes interior space of buildings but they lack features for the indoor space model, navigation network and semantic information. IndoorGML is a standard data model to store, represent, and exchange indoor spatial information and an XML application schema based on CityGML 3.2.1 (Kim et al., 2014). There are three aspects of IndoorGML including geometry of cells, network of cells and semantics of cells (see Figure 4).

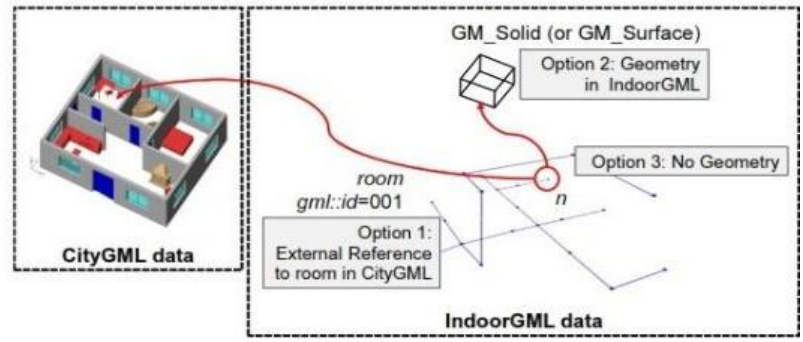

Figure 4. Geometry of cell of IndoorGML (Kim et al., 2014).

Representing the same 3D object (e.g. a building) with multiple representations produce some major drawbacks; redundancy of other less focused element (e.g. attribute and semantic, if focuses on geometry) and visualization (accessing only one model/LoD in a single viewer).

\subsection{Topology in Scale Modelling}

For many kinds of GIS applications such as emergency response applications, the interiors of the buildings need to be described along with the relative locations of the rooms, corridors, doors and exits, as well as their relationships to adjacent spaces. The relationship between adjacent spaces needs to be defined in a topological model. Topological modelling is a challenging task in the GIS environment, as the data structures required to express these relationships are particularly difficult to develop.

Topology is typically referring to relationship between spatial features/objects in enabling certain spatial functions such as network analysis. It also allows us to validate spatial dataset with certain predefined rules set by user/application (Kumar, 2016).

In representing real world objects, the term topology commonly refers to a mathematical approach that allows us to structure our dataset/model based on spatial relationship principles (e.g. Feature adjacency and connectivity) (Buckey, 2018).

Topological information/relationship can be assigned/retrieved based on user defined input (manual), predefined rules (sequences of inserting/converting data), geometrical connectivity, automatically generated (e.g. mathematically/fuzzy) and other in a computer.

In general, GIS topological modelling can be classified based on the following groups:

i. Matrix based

ii. Geometry based

iii. Topological primitives

iv. Graph based (e.g. node/edge navigation graph)

v. Semantic Topology (e.g. mathematical/fuzzy)

\subsection{Current Implementation}

LoD in 2D spatial can be seen in various approaches/model such as multi-scale, vario-scale (Meijers, 2011; Karim et al., 2016) or 2D generalization technique (see Figure 5).

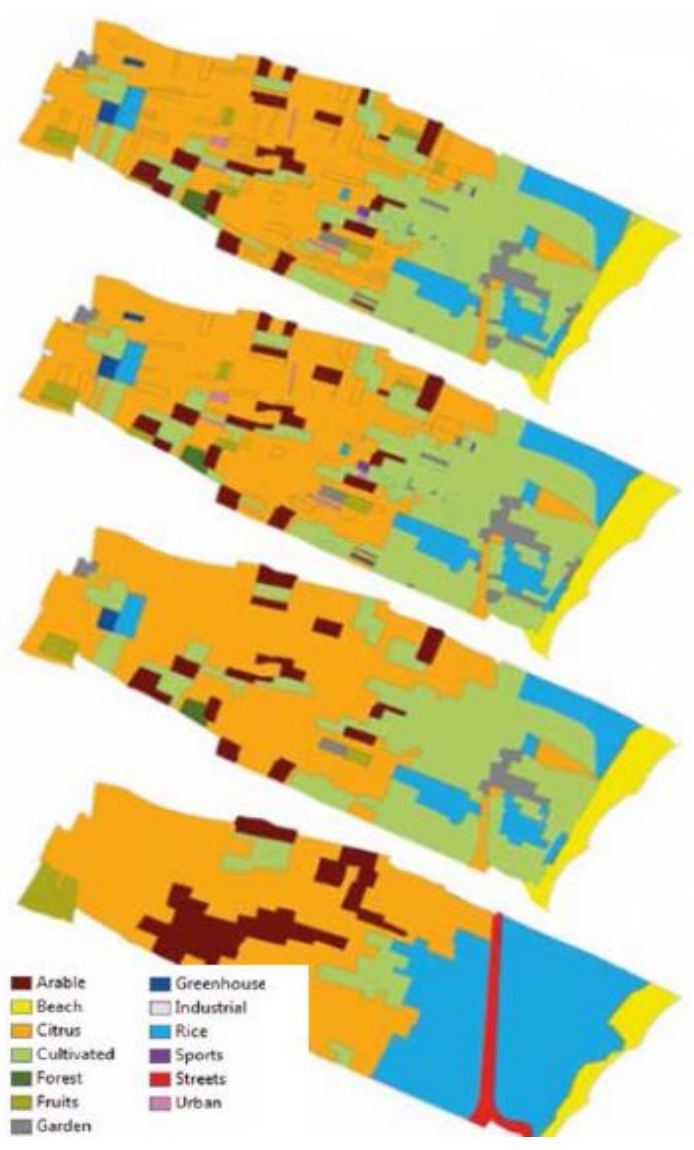

Figure 5. A series of automated generalization technique on land use by Dimov at el. (2014)

There are many researchers working on $2 \mathrm{D}$ and $3 \mathrm{D}$ data structure and data model. Most of them work toward a specific application, with only involve a level of detail, and for specific user. As for example, Dual Half Edge (DHE) data structure specially designed for indoor navigation application while utilizing internal and external topology (see Figure 6).

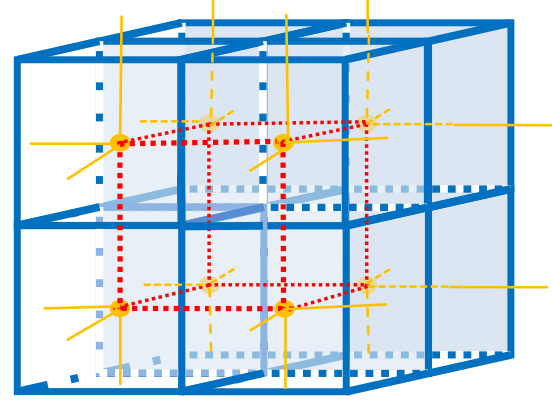

Figure 6. Primal and the dual spaces in a complex cell such as a building. 


\subsection{Drawback of Scale Dimension without Topology}

Different focus aspects or applications or users oriented customized models resulting difficulties in sharing the model within stakeholders. The current version of CityGML (v2.0) and next version (v3.0) are indeed useful for sharing the model and information with the same LoD. However, representing 3D objects (e.g. buildings) with multiple representations (LoDs) inevitably produce significant drawback; data redundancy, expensive in storage and visualization limitations (e.g. only one LoD per viewer) and disabling query from other LoD of the same object. Available GIS software solution either open source, commercial or customized application normally use single viewer to view each LoD. A new viewer will be opened if user want to compare the model/know the information (attribute) stored in other LoD.

Current research works indicate the need for a unified data model capable of supporting all aspects of geometry, attribute, semantic and topology. Designing a unified data model requires reliable data interoperability (module) for sharing 2D or 3D data across multiple scale models, applications and users (e.g. CityGML). Thus, in this research, a simplified topological model framework will be proposed to integrate information on multiple LoDs (CityGML) into a single viewer/selected model. Some topological concepts and database design will be discussed as the process in formulating the proposed idea.

\subsection{Previous Works}

Boguslawski et al. (2011) proposed a data structure called Dual Half-Edge (DHE) based on the Poincaré Duality. DHE data structure is used for 3D spatial modelling (see Figure 7). This structure resolves some of the modelling issues in 3D GIS and expresses the geometric structures as a cell complex, in preserving adjacency relationships between cells, and including semantic information using attributes.

The DHE is based on the quad-edge and its extension - the augmented quad-edge (Ledoux and Gold, 2007). These structures allow the construction of models and their duals at the same time. Dual space is used to connect cells in a complex and to navigate between them. Other data structures such as the half-edge and winged-edge do not provide information for management of the duality. The construction process using the DHE is based on Euler operators which are used widely in Computer Aided Design (CAD) systems for B-Rep modelling. They preserve the topological consistency of the model at each step of a construction process.

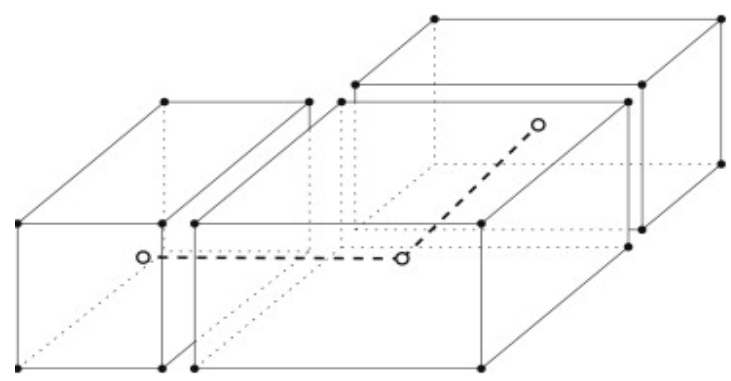

Figure 7. The DHE data structure models 3D subdivisions by representing the boundary of each polyhedron separately with a graph (solid lines), and two adjacent polyhedra are linked together by the dual graph (dashed lines). Both graphs are interconnected (Boguslawski et al., 2011).
2D-to-3D scale generalization technique derived from combination of simplified dual half edge data structure with vario-scale approach has been tested by Karim et. al. (2016). This technique produces the hierarchical connection between lower and upper details level which capable to access the geometry topology, attributes and semantic information across the same object (with differ LoD) and neighbour objects Figure 8. From 2D most detailed dataset, a number of $N-1$ variable scale LoDs dataset could be generated via extrusion (by point, line and polygon) and slicing technique.
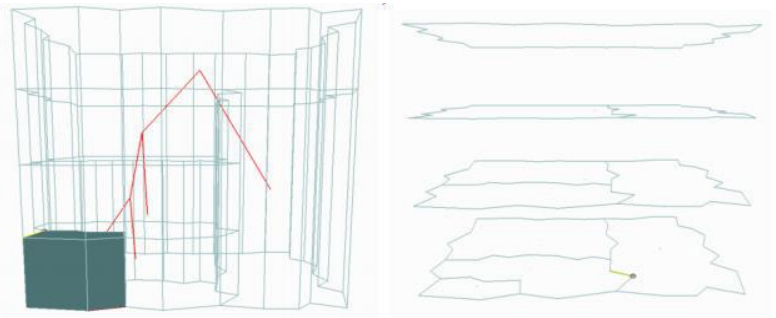

Figure 8. 2D modelling with scale as 3rd dimension and topologically connected using hierarchical graph.

Other derived structure from DHE is designed for rapid indoor navigational network, a proposed simplified structure was developed to support indoor navigation network using topological graph. Simplified data model by Jamali et al. (2017) includes geometrical, semantic and topological model (Figure 9 and 10). Topological model is a graph which is used for spatial queries such as shortest path finding between geometrical elements (e.g. rooms) (see Figure 10). Indoor building elements are represented by geometrical model which are topologically connected in topological space and properties of each building element is stored as semantic information.

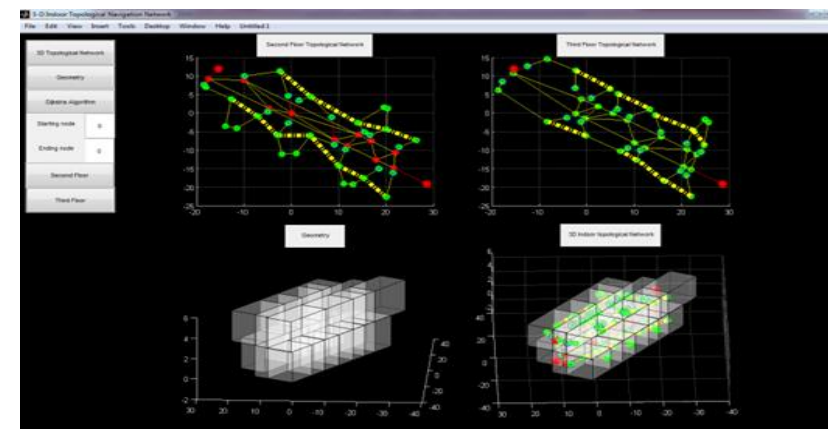

Figure 9. Rapid indoor data structure and navigational network.

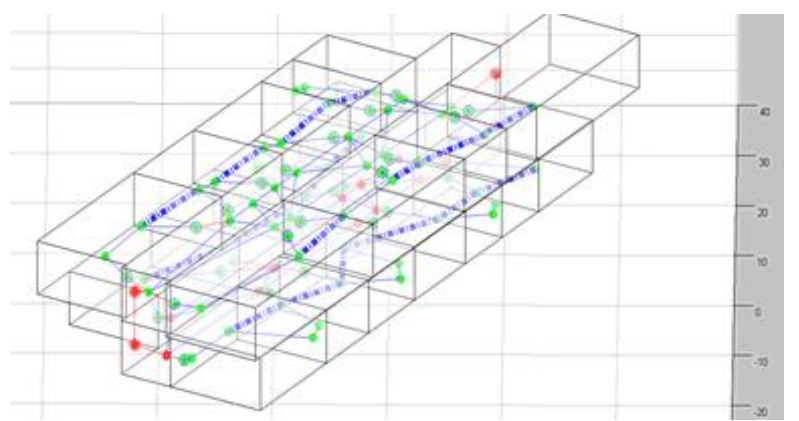

Figure 10. Simplified data model by Jamali et al. (2017). 


\section{PROPOSED UNIFIED TOPOLOGY IN MULTI- SCALE MODELLING}

The proposed framework on unifying topological, semantic and geometry of the object should cover the following aspects:

- $\quad 2 \mathrm{D}$ and 3D model integration

- Multiple LoDs (e.g. CityGML Standard - LoD0LoD4)

- Topology connected with the geometry and associated with the semantic information.

- Provide navigation procedure either using geometry, topology or attribute query.

- Without generalization technique (miss out the details info/data at detailed level)

- Connected using database (faster query)

- $\quad$ Single viewer (either LoD1, 2 or 3)

The initial concept is based on the Poincaré duality as illustrated in Figure 11 and then being simplified only to interior or cell dual (remove any vertex/edges outside the model toward universal edges). Later, it is re-modified to support the scale implementation using hierarchical tree structure (only for dual graph - vertex and edge) in storing attribute and navigational order/steps, e.g. predecessor and successor (Figure 13). Each building will be assigned a global vertex and multiple edges in connecting their respective dual graph in this simplified topological model (Figure 12). A graph consisting of nodes and edges in which nodes represent the position or location of an object such as a room while edges represent connection between nodes.

Later, this topological graph table in Figure 14 will be simplified into a single dual node as for representing the unique ID/spatial for the object (2D or 3D as a block) at any LoD. All LoDs (the same object) will be stored in a 3D spatial database (e.g. 3D Oracle Spatial) to be visualized in a single selected LoD as illustrated in Figure 13.According to the proposed general concept (Figure 12), an efficient flow of storing the data (topology and semantic) within a spatial database should be created. Proposed table structure for navigation graph (geometry and topology) between 2D and 3D objects (neighbours) can be implemented as in Figure 13 and Figure 14.

Any queries related toward information from other LoDs attributes will activate the spatial ID of global vertex/edge of a particular LoD and get access to the topological/geometrical graph in the same level. Information/attribute stored in topological or geometrical nodes, edges, faces or 3D cells can be accessed from Viewer to database via the connector and relationship within the proposed framework (Figure 15).

Simple geometry (e.g. a room)

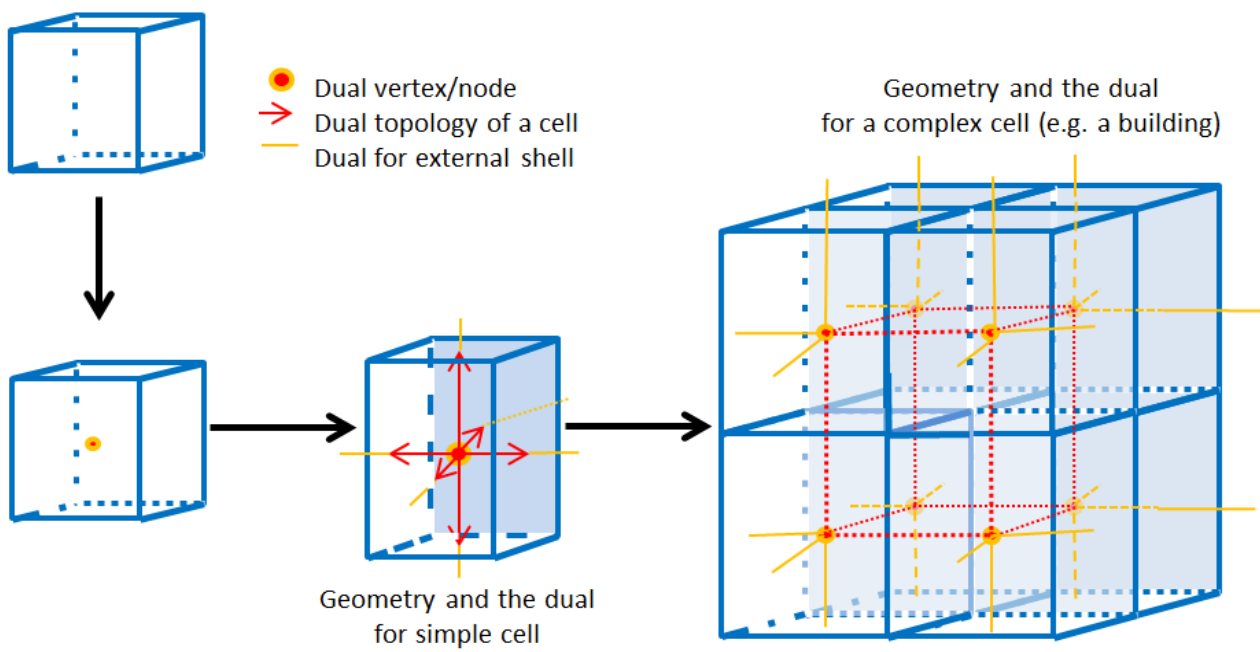

Figure 11. Poincaré duality derived concept.
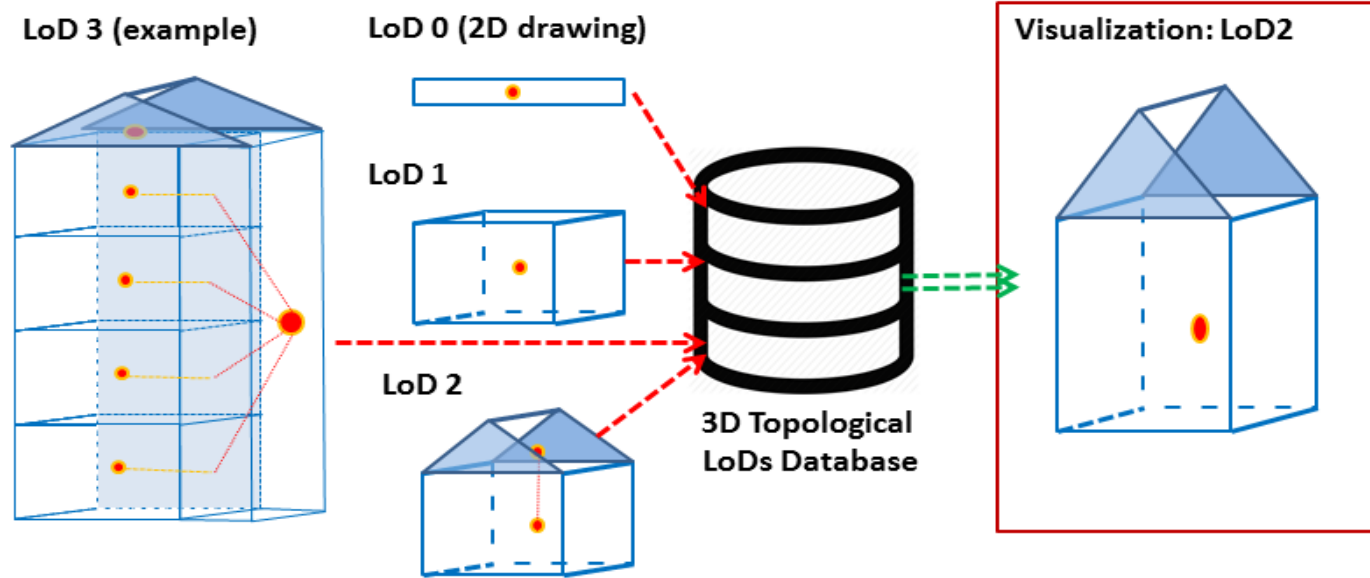

Figure 12. Simple illustration of overall topological graph 


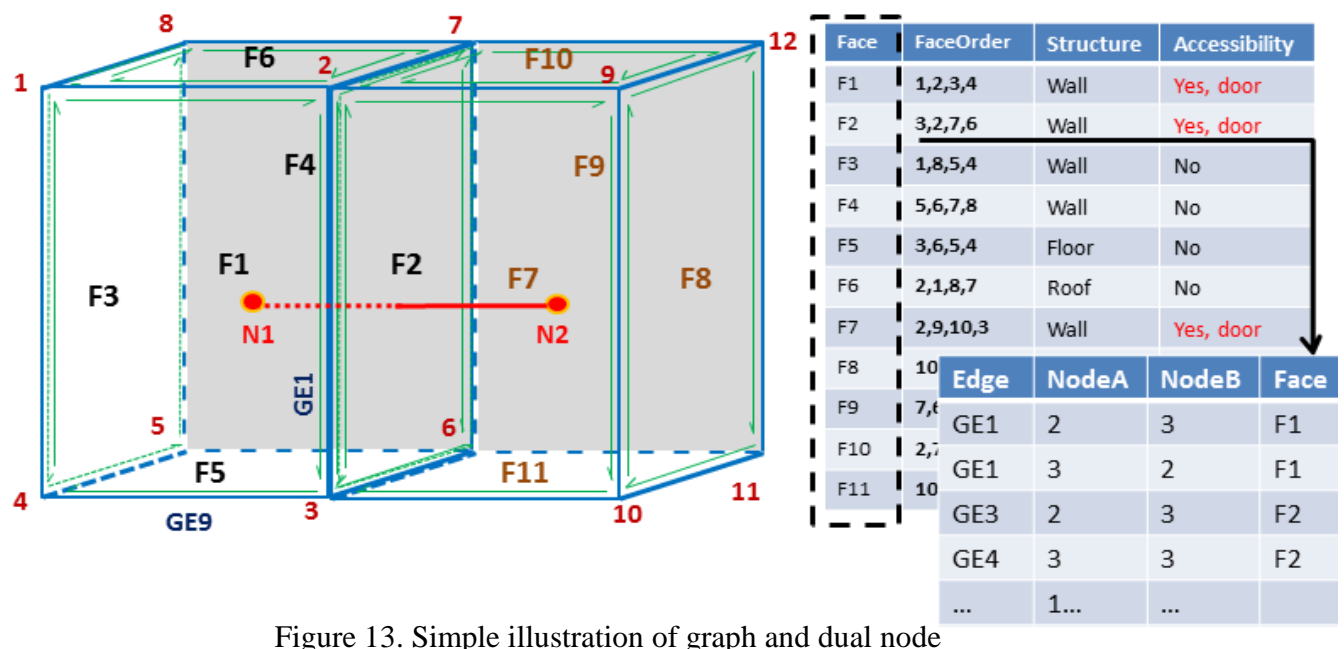

Figure 13. Simple illustration of graph and dual node

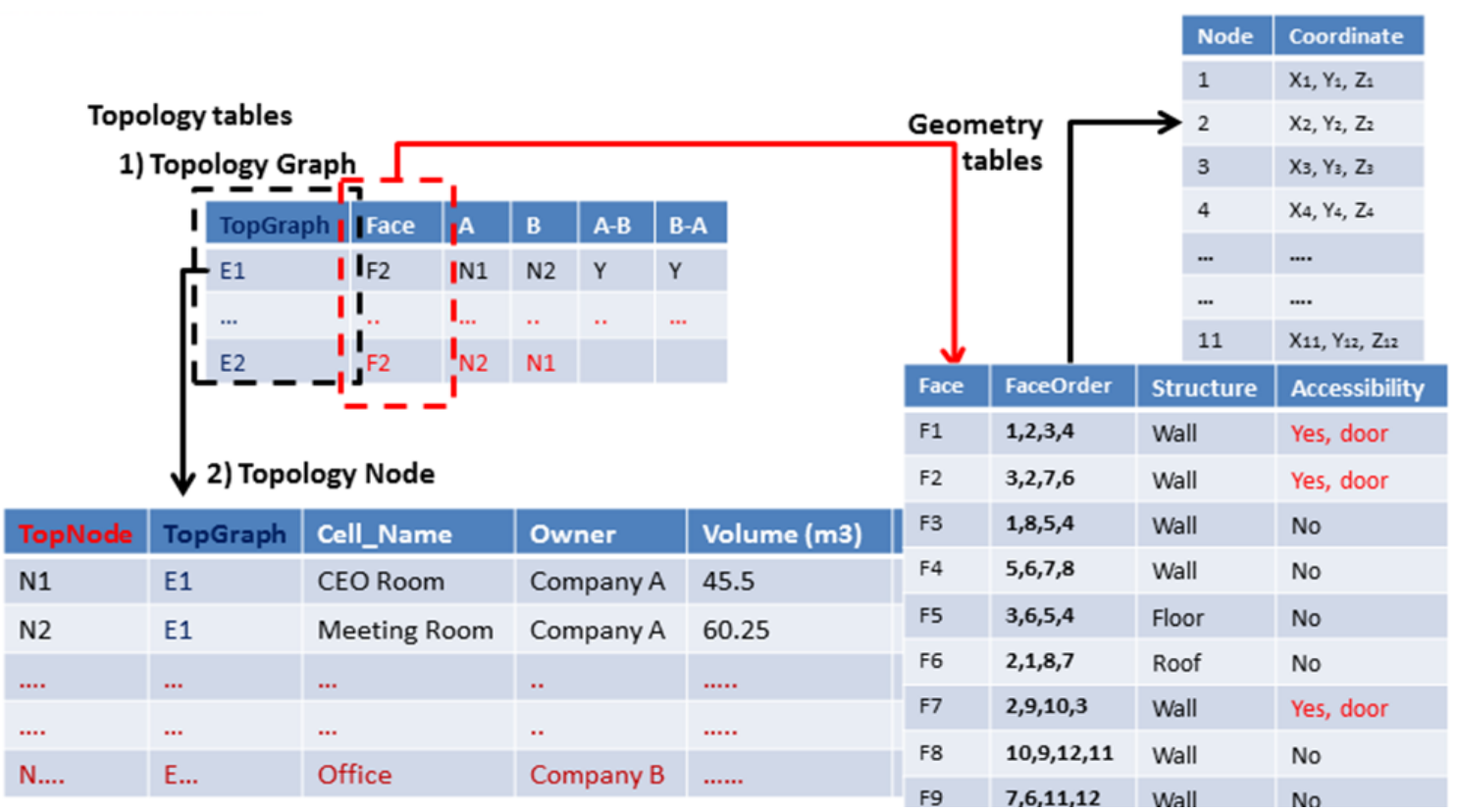

Figure 14. Relationship of geometry, graph (topology) and attribute table.

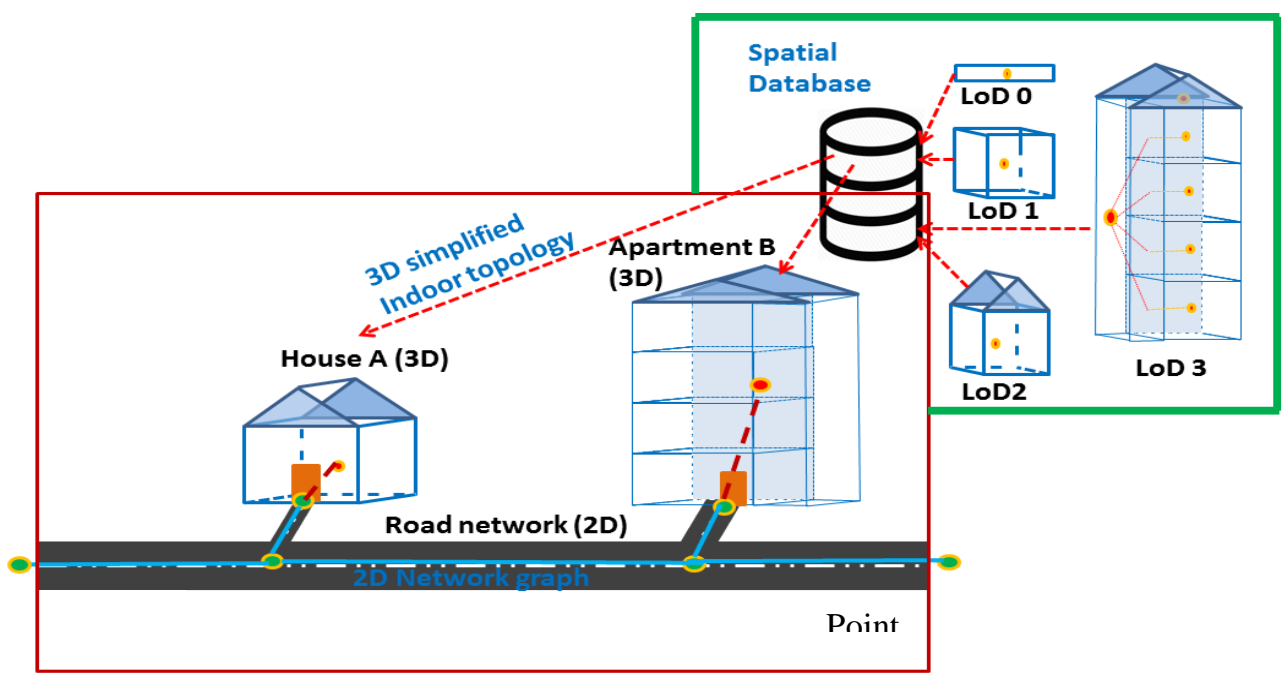

Figure 15. Simple illustration of overall processes either in visualization (viewer) or database (backend storing attribute with topological graph) 


\section{POTENTIAL APPLICATIONS}

\subsection{Utility Mapping (Underground and in Building block)}

Underground pipeline/gas network naturally will be model using single/actual dimension such as in Figure 16. However, this single dimension of underground utility or road network can be integrated with multiple LoD of the building in distribution pipe (as illustrated in Figure 17). Examples of scenarios are the apartment and huge block of complex building - shopping mall.
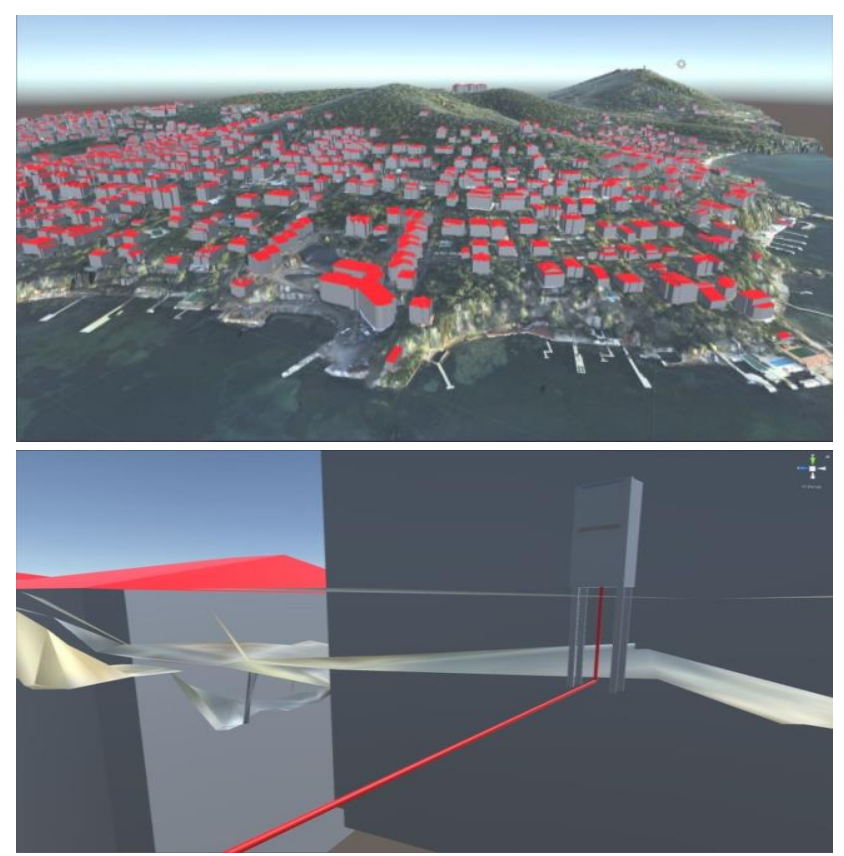

Figure 16. Example of utility mapping by BIMTAS company.

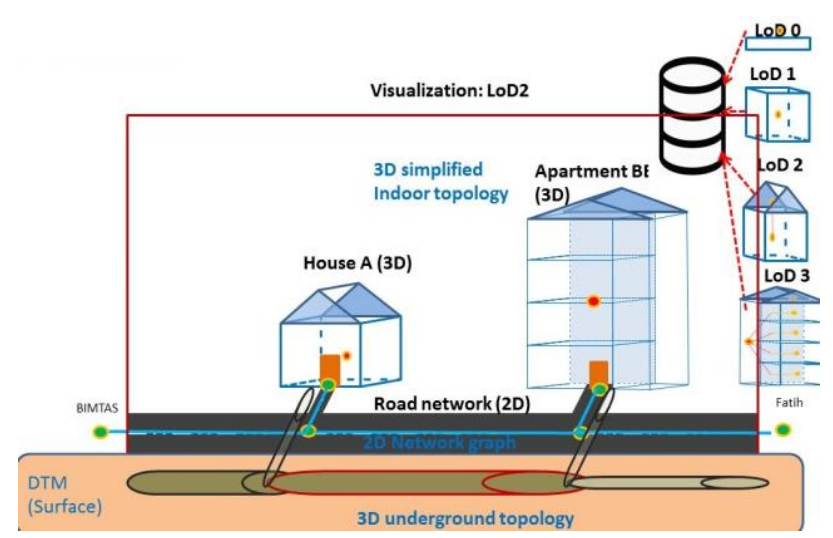

Figure 17. Illustration on unify topological modelling for utility (single scale, road -2 LoDs, and bulding 3 LoDs.

Example of Pseudo/conceptual flow

Block selection (in Viewer, e.g. Building \& Pipeline in LoD1) $\rightarrow$ query on LoD info (e.g. 2 LoD options, selection LoD2) $\rightarrow$ select/query on the pipe network $\rightarrow$ extract info (estimated solar energy in LoD3) to the Viewer (LoD1).

\subsection{Estimating Solar Panel}

Since calculation of the potential estimated solar energy of a building (façade or/and roof) closely influenced by the use of LoD building model (e.g. LoD1, LoD2 and LoD3), information on the amount of estimated energy could be stored in each LoD in a pre-execute LoD model. Daily, weekly, monthly and yearly basis (Figure 18 and 19) query can be perform for each LoD and comparison on which LoD is the best based on the actual collected energy.

Amount will be slightly differing from each LoD, somehow we may retrieve this information even using LoD1 (simple bounding box in presenting a building) in a visualization viewer. Thus, geometry of rendering time for a big city become faster in and may support more buildings.

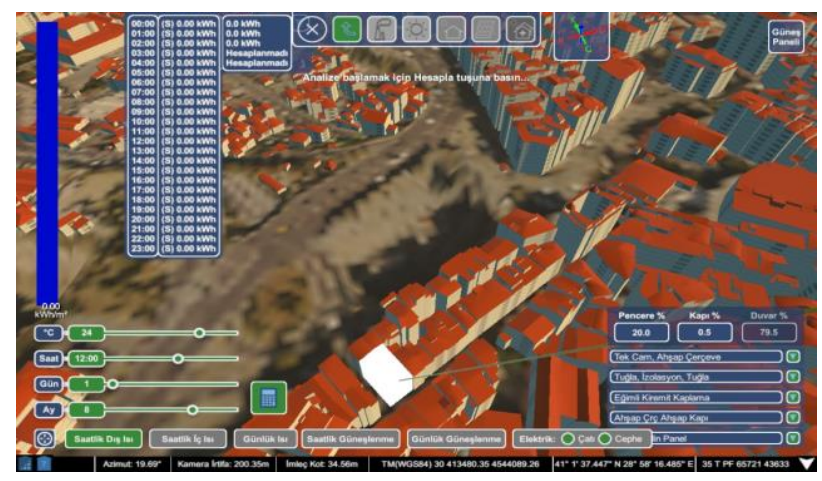

Figure 18. Selection of a particular building to be calculated, single LoD.

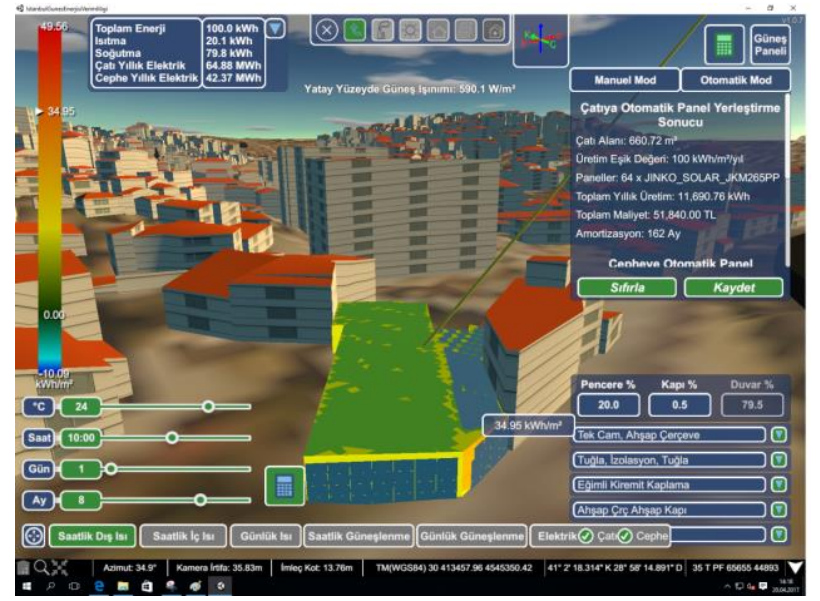

Figure 18. Solar estimation for the selected building rooftop and façade (potential solar panel).

Example of Pseudo/conceptual flow Block selection (in Viewer, e.g. LoD1) $\rightarrow$ query on LoD info (e.g. 3 LoD options, selection LoD3) $\rightarrow$ select/query on roof or/and façade of pre-executed model $\rightarrow$ select date/time in the selected LoD info $\rightarrow$ extract info (estimated solar energy in LoD3) to the Viewer (LoD1). 


\section{CONCLUSION}

In this paper, we presented a brief literature on 2D and 3D scale with topological modelling and described some limitations. The motivation of this research is to solve current multirepresentation model drawback especially on a single visualization and attribute, without make use/retrieve of information from other LoD model - redundancy. We proposed a conceptual framework towards unifying multiple level of 3D model via topological connectivity in a single viewer. Information from other LoDs can be retrieved to support current-future analysis/applications. The proposed concept should be able to access semantic, attribute and geometry information via topological graph across LoDs. We also believe that this method provides less storage consumption, graphic and time required for rendering the model. It produces a flexibility and simple to integrate with other $2 \mathrm{D}$ and $3 \mathrm{D}$ data structure. The model also should be able to integrate multiple LoDs (Geometry) for semantic and information extraction.

\section{ACKNOWLEDGEMENT}

BIMTAS Company, Istanbul, Turkey.

\section{REFERENCES}

Baig, S.U., Hassan, M.I. and Rahman, A.A., 2011. Automatic Generalization of 3D Building Models - A Review, 10th International Symposium \& Exhibition on Geoinformation (ISG 2011). ISPRS, Shah Alam Convention Centre(SACC), Malaysia.

Boguslawski, P., Gold, C.M. \& Ledoux, H., 2011. Modeling and analysing 3D buildings with a primal/dual data structure. ISPRS Journal of Photogrammetry and Remote Sensing, 66(2): 188-197.

Buckey, D. J., NILS.GIS Introduction - Chapter 2 (Topology). http://planet.botany.uwc.ac.za/nisl/gis/gis_primer/page_22.htm [Cited: April 4, 2018].

Dimov D., Palomar J.M., Ruiz A.L. . 2014. Automated Generalization of Land-Use Data with GIS-Based Programming

Emgård L., Zlatanova S., 2008. Implementation alternatives for an integrated 3D Information Model. In: van Oosterom P., Zlatanova S., Penninga F., Fendel E.M. (eds) Advances in 3D Geoinformation Systems. Lecture Notes in Geoinformation and Cartography. Springer, Berlin, Heidelberg.

Groger, G., and Plumer, L. , 2011. How to achieve consistency for 3D city models. Geoinformatica, 15(1), 137-165.

Jamali, A., Abdul Rahman, A., Boguslawski, P., Kumar, P., \& M. Gold, C. (2017). An automated 3D modeling of topological indoor navigation network. GeoJournal. 82(1). 157-170.

Kolbe, T. H., Groher, G., \& Plumenr, L., 2008. CityGML - 3D city models and their potential for emergency response. Geospatial information technology for emergency response, 257.

Kim, J. S., Yoo, S. J., \& Li, K. J. Integrating IndoorGML and CityGML for indoor space. In International Symposium on Web and Wireless Geographical Information Systems. (2014, May). (pp. 184-196). Springer Berlin Heidelberg.

Kwan M. P. and Lee J., 2005. Emergency response after 9/11: the potential of real-time 3D GIS for quick emergency response in microspatial environments. Computers, Environment and Urban Systems 29(2):93-113
Karim, H., Rahman, A. R., and Boguslawski, P., 2016. ISPRS Volume XLII-2/W1, 2016. $3^{\text {rd }}$ International GeoAdvances Workshop, 16-17 October 2016, Istanbul, Turkey.

Kumar, R., 2016. Topology in GIS . Online slide 2016. https://www.slideshare.net/RohitKumar431/topology-in-gis [Cited: April 24, 2018].

Ledoux, H. and Gold, C.M., 2007. Simultaneous storage of primal and dual three-dimensional subdivisions. Computers, Environment and Urban Systems, 31(4): 393-408.

Meijers, M., 2011. Variable-Scale Geo-Information, PhD Thesis. Technische Universiteit Delft, Netherlands.

\section{Revised August 2018}

